\title{
LETTER \\ Iris Recognition Based on Local Gabor Orientation Feature Extraction
}

\author{
Jie SUN ${ }^{\dagger}$, Lijian ZHOU ${ }^{\dagger}$, Nonmembers, Zhe-Ming $\mathrm{LU}^{\dagger \dagger}{ }^{\dagger a}$, Member, and Tingyuan $\mathrm{NIE}^{\dagger}$, Nonmember
}

\begin{abstract}
SUMMARY In this Letter, a new iris recognition approach based on local Gabor orientation feature is proposed. On one hand, the iris feature extraction method using the traditional Gabor filters can cause timeconsuming and high-feature dimension. On the other hand, we can find that the changes of original iris texture in angle and radial directions are more obvious than the other directions by observing the iris images. These changes in the preprocessed iris images are mainly reflected in vertical and horizontal directions. Therefore, the local directional Gabor filters are constructed to extract the horizontal and vertical texture characteristics of iris. First, the iris images are preprocessed by iris and eyelash location, iris segmentation, normalization and zooming. After analyzing the variety of iris texture and 2D-Gabor filters, we construct the local directional Gabor filters to extract the local Gabor features of iris. Then, the Gabor \& Fisher features are obtained by Linear Discriminant Analysis (LDA). Finally, the nearest neighbor method is used to recognize the iris. Experimental results show that the proposed method has better iris recognition performance with less feature dimension and calculation time.

key words: iris recognition, texture orientation, Gabor filters, linear discriminant analysis, feature extraction
\end{abstract}

\section{Introduction}

Identity recognition based on irises is the most promising technique in high-security environments among various biometric features such as face, fingerprint, palm vein, signature and palm print. The reason is that the iris has unique, stable and non-invasive characteristics. A number of scholars have paid much attention to iris recognition. Liu and Xie [1] adopted the Discrete Linear Discriminant Analysis (DLDA) to extract iris features and then used the Euclidean distance to match the features. Patil et al. [2] analyzed and compared three kinds of iris recognition methods based on PCA, Log_Gabor and Gabor wavelets respectively. Ahamed and Bhuiyan [3] proposed a low-complexity iris recognition method based on the Curvelet transform domain. Rajput and Waghmare [4] adopted the Contourlet transform to extract iris features. Yuan et al. [5] adopted the 2D-Gabor filters to extract the iris features. This method paid much attention to the selection of 2D-Gabor filters' directions. Sun et al. [6] adopted the Curvelet transform to extract the iris feature, where the iris Curvelet features are mapped by PCA

Manuscript received September 19, 2014.

Manuscript revised February 6, 2015.

Manuscript publicized April 22, 2015.

${ }^{\dagger}$ The authors are with School of Communication and Electronic Engineering, Qingdao Technological University, Qingdao 266033 PR China.

${ }^{\dagger}$ The author is with School of Aeronautics and Astronautics, Zhejiang University, Hangzhou 310027, China.

a) E-mail: zheminglu@zju.edu.cn (Corresponding author) DOI: $10.1587 /$ transinf.2014EDL8188 and LDA to further extract the features. Ng et al. [7] proposed an iris recognition system based on a basic and fast Haar wavelet decomposition method to extract the iris feature. In this method, they decomposed the iris region using Haar wavelet into four levels. And only the fourth level coefficients are converted to the binary iris code. Finally, the Hamming distance is used for recognize.

In order to sufficiently consider the iris texture orientation characteristics and better improve the high feature dimensions, this paper proposes an iris recognition method based on local Gabor orientation feature extraction. First, the iris images are preprocessed by iris and eyelash location, iris segmentation, normalization and zooming. After analyzing the variety of iris texture orientation and directional selection of 2D-Gabor filters, we construct the local Gabor filters with orientation feature extraction. Next, we use the local Gabor filters to extract the iris local Gabor features. And then the Gabor \& Fisher features are obtained by LDA. Finally, the nearest neighbor method is used to recognize the iris. Experimental results show that the proposed method has better iris recognition performance with fewer feature dimensions and calculation time.

\section{Iris Image Pre-Processing}

A captured iris image contains not only the region of interest (iris) but also some 'unuseful' parts (e.g. eyelid, pupil etc.). Thus, the iris image cannot be used directly without preprocessing. In addition, the iris position in the iris image will change for different acquisition environments. The iris elastic deformation caused by nonuniform illumination and mood swings will cause the changes in the size and the eyelash shading will influence the iris reorganization performance as well. Therefore, the iris pre-processing of iris and eyelash location, iris segmentation, normalization and zooming are needed before iris feature extraction.

We take an iris image with a resolution of $480 \times 640$ from CASIA-Iris-Syn of the CAISA-IrisV4 iris dataset for example as shown in Fig. 1 (a). The iris is an annular area located between pupil (inner boundary) and sclera (outer boundary). Since the iris inner boundary is with a large gray gradient, the pupil can be separated by the thresholding method. The outer boundary can be detected with the Canny operator [8] and the results are shown in Fig. 1 (b). From Fig. 1 (b), we can see that the eyelash and eyelid are contained in the segmented iris area, which will influence the recognition performance. In this Letter, the Sobel fil- 


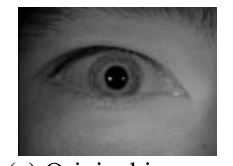

(a) Original image

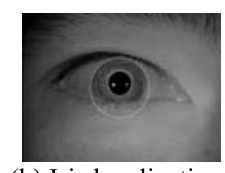

(b) Iris localization

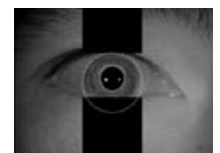

(c) Eyelid localization

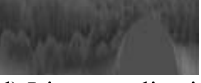

(d) Iris normalization (e) Image zooming ter and Order-Statistic filter are used to remove the eyelash and eyelid respectively [9] and the results are shown in Fig. 1 (c). Considering the annular characteristics of the iris, we use the polar coordinate system of Daugmn's [8] normalization model to normalize the iris and the result is shown in Fig. 1 (d) with the image size of $100 \times 240$. Then we reduce the image into the size of $50 \times 120$ pixels and the result is shown in Fig. 1 (e).

\section{Construction of Local Gabor Filters}

\subsection{Iris Gabor Features}

In general, the detailed description of 2-D Gabor filters can be found in [10]. The amplitudes of traditional Gabor filters with 5 scales and 8 orientations [11] are shown in Fig. 2 and we can see that the 2D Gabor filters have advantages in orientation selection. We take a preprocessed iris image in Fig. 1 (e) as example that is filtered by traditional Gabor filters to get 40 Gabor feature images with a resolution of $50 \times 120$. The amplitudes of Gabor feature images are shown in Fig. 3, where the every row denotes the different scale and every column denotes the different orientation. In this letter the amplitudes are extracted as Gabor features. If we make use of the 40 iris Gabor images to form the iris feature vector directly, the feature dimension is up to $50 \times 120 \times 40=240000$ that is larger than 6000 (the size of the original image). In order to reduce the feature dimension and feature extraction time, we choose the Gabor filters with specific orientations to form the local Gabor filters according to the change characteristics of the iris texture and the performance of Gabor filters in every orientation.

\subsection{Analysis of Iris Texture Characteristics}

From the original image as given in Fig. 1 (a), we can see that the changes of iris textures in angle and radial directions are more obvious than in other directions and these changes will reflect in the vertical and horizontal directions of the preprocessed image as given in Fig. 1 (e). To observe the iris texture changes, we randomly take 4 persons and 5 images per person from the iris dataset as the example to analyze the iris texture changes and the original images are shown in Fig. 4. From Fig. 4, we can see that the changes of original iris texture in angle and radial directions are more obvious than the other directions. The preprocessed images are shown in Fig. 5. From Fig. 5, we know that the changes

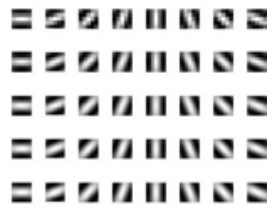

Fig. 2 Amplitudes of traditional Gabor filters

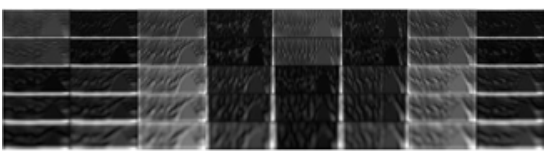

Fig. 3 Iris Gabor features

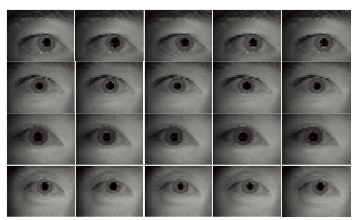

Fig. 4 Localized iris images of different persons

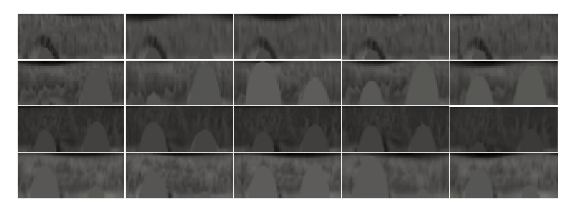

Fig. 5 Normalized iris images of different persons

mentioned above reflect in the vertical and horizontal directions. Therefore, we select filters in these specific directions to construct the local orientation Gabor filters as described in Sect. 3.3. And then we use the local orientation Gabor filters to extract the iris Gabor feature.

\subsection{Gabor Filters Orientation Performance Analysis}

From Fig. 3, we find that the extracted iris texture feature will change when the Gabor filter selects different orientations. If the Gabor filters are built by choosing the filters corresponding to the filers with larger response of the iris texture in vertical direction and horizontal orientation, the better recognition performance will be achieved. Therefore, we choose randomly 30 persons and 10 images per person to preprocess. And then 5 pre-processed images with a resolution of $50 \times 120$ of one person are selected as the training set, the rest as the testing set. We use the Gabor filters in every orientation to extract the preprocessed image respectively and compare the recognition performance, the results are shown in Table 1. From Table 1, we know that the recognition rate is maximum when the iris features are extracted by the vertical filters $(\pi / 2)$, following by near vertical filters $(3 \pi / 8,5 \pi / 8)$ and horizontal filters $(0, \pi)$. The results are same as the changes of the iris texture characteristics analyzed in above section. So, we choose 6 orientations namely $\{4 \pi / 8,5 \pi / 8,3 \pi / 8,2 \pi / 8,0,7 \pi / 8\}$ corresponding to the highest recognition rate in Tables 1 and 5 scales to construct the 
Table 1 Iris recognition rate of the Gabor filtesr in one direction

\begin{tabular}{|c|c|c|c|}
\hline Filter direction & Recognition rate (\%) & Filter direction & Recognition rate (\%) \\
\hline 0 & 68.89 & $4 \pi / 8$ & 76.30 \\
\hline$\pi / 8$ & 67.31 & $5 \pi / 8$ & 74.81 \\
\hline $2 \pi / 8$ & 69.63 & $6 \pi / 8$ & 65.19 \\
\hline $3 \pi / 8$ & 73.33 & $7 \pi / 8$ & 67.41 \\
\hline
\end{tabular}

Table 2 Feature extraction time of different Gabor filters

\begin{tabular}{|c|c|c|c|}
\hline Gabor filters & The number of filters & Feature dimension & $\begin{array}{c}\text { Feature extraction } \\
\text { time/ms }\end{array}$ \\
\hline Gabor1 & 30 & 180000 & 92.1 \\
\hline Gabor2 & 40 & 240000 & 126 \\
\hline
\end{tabular}

local orientation Gabor filters that are denoted as Gabor1.

The preprocessed images with a resolution of $50 \times 120$ are extracted by traditional Gabor filters (denoted as Gabor2) with 5 scales and 8 orientations and proposed Gabor 1 respectively, the feature dimensions and feature extraction time are shown in Table 2. We can see that the feature dimensions extracted by Gabor 1 decrease by $25 \%$ compared to that extracted by Gabor2. In addition, the proposed Gabor1 shortens the feature extraction time greatly.

\section{Iris Recognition Based on Local Gabor Orientation Feature Extraction}

We propose the iris recognition approach based on local Gabor orientation feature extraction due to the iris texture consisting of some directional textures such as crypt, fold and pigment spots. The flow chart is shown in Fig. 6. First, the iris images are preprocessed by iris and eyelash location, iris segmentation, normalization and zooming. After analyzing the variety of iris texture orientation and directional selection of 2D-Gabor filters, we construct the local Gabor filters with orientation feature extraction. Next, we use the local Gabor filters to extract the iris local Gabor features. The Gabor \& Fisher features are then obtained by LDA. Finally, the nearest neighbor method is used to recognize the iris. Now we illustrate the proposed algorithms as follows.

Step 1. Preprocess all training images $\mathbf{X}_{1}, \mathbf{X}_{2}, \ldots, \mathbf{X}_{N}$ by iris localization, eyelid localization, normalization and zooming.

Step 2. Select 30 filters with 5 scales and 6 orientations $\{4 \pi / 8,5 \pi / 8,3 \pi / 8,2 \pi / 8,0,7 \pi / 8\}$ to construct the Gabor filters with orientation feature extraction according to the variety of iris texture orientation and directional selection of 2D-Gabor filters.

Step 3. Use the Gabor filters constructed in Step 2 to extract the iris texture directional characteristics and get the iris local Gabor subimages. Reshape every subimage to a row vector and then combine them to form a row vector $Y_{i}(i=1,2, \cdots, N)$ one by one.

Step 4. Transform the $Y=\left[Y_{1}, Y_{2}, \cdots Y_{N}\right]$ by LDA to get the Fisher feature sub space $K$.

Step 5. Project the feature vector $Y_{i}$ on the Fisher feature sub space $K$ to get Gabor \& Fisher features.

Step 6. Extract the preprocessed testing images by local

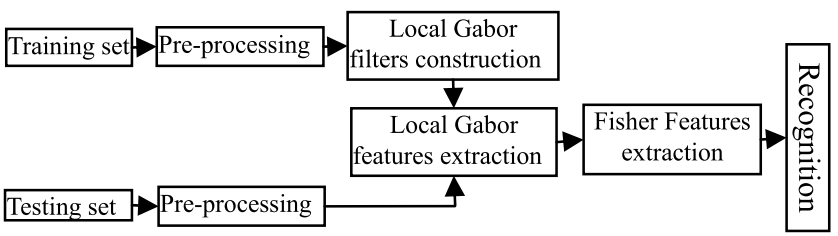

Fig. 6 Proposed algorithm flow chart

Gabor filters constructed in Step 2 to get the iris texture directional characteristics.

Step 7. Project the testing samples on the Fisher feature sub space $\mathrm{K}$ to get the Gabor \& Fisher features of the testing samples.

Step 8. Classify the iris by the nearest neighbor method, which realizes the proposed approach "Gabor1+LDA".

\section{Experimental Results and Analysis}

In order to test the performance of the proposed method, this letter adopts CASIA-Iris-Syn and CASIA-Iris-Lamp of the CASIA-V4 iris dataset to perform the comparison experiments. We randomly select 50 persons, 10 images per person with 256 grayscales from the iris database. For convenience, our experiments adopt the iris image of size $50 \times 120$ after preprocessing. We randomly take $n$ images from every person as the training set, the others as the testing set in every experiment. We take the Average Recognition Rate (ARR) and Variance (VR) of the iris recognition rate as the assessment criterion to evaluate the proposed approach. Generally, the smaller inner-class distance is, the better classification effect is. Therefore, the Average Minimum Classification Distance (AMCD) and Variance (VR) between the recognized test samples and the trainning samples are computed to further evaluate the performance of proposed method. For comparison with other methods, we do the experiments on every database using Gabor2+LDA, Curvelet+PCA+LDA (CPL) [6] and Haar decomposition (Haar) [7] respectively.

\subsection{Experiment on CASIA-Iris-Syn Database}

CASIA-Iris-Syn contains 10,000 synthesized iris images of 1,000 classes, each having 10 iris images and the size of each image is $480 \times 640$. The irises' textures of CASIA-IrisSyn are synthesized automatically from a subset of CASIAIirsV1. In this database, the intra-class variations are introduced into the synthesized iris dataset including deformation, blurring and rotation. The example images of 2 persons, 5 images per person are shown in Fig. 7. The recognition results are shown in Table 3 and the results of AMCD and VR are shown in Table 4.

From Table 3, we can see the performance of the proposed method based on local Gabor orientation feature extraction is better. The recognition rate is similar to Gabor2+LDA but the speed of the proposed method is quicker than the Gabor2+LDA method. The highest average 


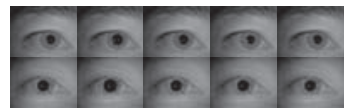

Fig. 7 Iris-Syn database

Table 3 Recognition results on the Syn iris database

\begin{tabular}{|c|c|c|c|c|c|c|c|c|}
\hline \multirow{2}{*}{$\mathrm{n}$} & \multicolumn{2}{|c|}{ Gabor1+LDA } & \multicolumn{2}{c|}{ Gabor2+LDA } & \multicolumn{2}{c|}{ CPL } & \multicolumn{2}{c|}{ Haar } \\
\cline { 2 - 9 } & ARR & VR & ARR & VR & ARR & VR & ARR & VR \\
\hline 1 & 26.31 & 0.06 & 25.33 & 0.16 & 27.33 & 0.71 & 24.82 & 0.41 \\
\hline 2 & 70.05 & 0.60 & 45.23 & 0.25 & 75.00 & 0.21 & 64.35 & 0.22 \\
\hline 3 & 85.71 & 0.10 & 63.07 & 1.57 & 79.89 & 1.23 & 76.16 & 1.73 \\
\hline 4 & 88.40 & 0.19 & 77.22 & 1.74 & 85.93 & 1.15 & 81.48 & 2.14 \\
\hline 5 & 89.26 & 0.17 & 84.91 & 4.61 & 88.23 & 1.52 & 84.44 & 1.57 \\
\hline 6 & 93.24 & 0.06 & 91.76 & 1.29 & 90.24 & 2.05 & 88.89 & 0.89 \\
\hline 7 & 94.53 & 0.08 & 95.06 & 0.16 & 93.21 & 0.76 & 91.67 & 1.67 \\
\hline 8 & 97.78 & 0.11 & 97.96 & 0.11 & 96.30 & 1.90 & 94.44 & 1.46 \\
\hline 9 & 98.87 & 0.06 & 98.82 & 0.20 & 96.30 & 1.43 & 96.30 & 0.85 \\
\hline
\end{tabular}

Table 4 AMCD and VR on the Syn iris database

\begin{tabular}{|c|c|c|}
\hline Different methods & AMCD & VR \\
\hline Gabor1+LDA & 0.1198 & 0.0170 \\
\hline Gabor2+LDA & 0.1953 & 0.0204 \\
\hline CPL & 0.4589 & 0.1581 \\
\hline Haar & 0.2715 & 0.2123 \\
\hline
\end{tabular}

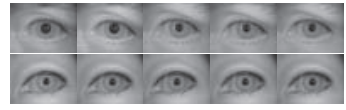

Fig. 8 Iris-Lamp database

Table 5 Recognition results on the Lamp iris database

\begin{tabular}{|c|c|c|c|c|c|c|c|c|}
\hline \multirow{2}{*}{$\mathrm{n}$} & \multicolumn{2}{|c|}{ Gabor1+LDA } & \multicolumn{2}{c|}{ Gabor2+LDA } & \multicolumn{2}{c|}{ CPL } & \multicolumn{2}{c|}{ Haar } \\
\cline { 2 - 9 } & ARR & VR & ARR & VR & ARR & VR & ARR & VR \\
\hline 1 & 27.78 & 0.03 & 25.39 & 0.15 & 29.17 & 0.54 & 10.57 & 2.81 \\
\hline 2 & 63.21 & 0.20 & 64.23 & 0.59 & 65.78 & 0.17 & 23.46 & 1.57 \\
\hline 3 & 71.43 & 0.21 & 75.89 & 2.19 & 78.31 & 1.23 & 70.37 & 4.10 \\
\hline 4 & 86.42 & 0.13 & 85.31 & 0.07 & 85.19 & 0.36 & 76.53 & 2.25 \\
\hline 5 & 91.11 & 0.10 & 86.44 & 4.08 & 88.90 & 2.23 & 84.44 & 1.32 \\
\hline 6 & 95.06 & 0.22 & 94.53 & 0.32 & 91.11 & 0.78 & 90.12 & 0.42 \\
\hline 7 & 97.90 & 0.04 & 97.82 & 0.13 & 92.59 & 0.96 & 93.83 & 0.23 \\
\hline 8 & 98.47 & 0.05 & 9.52 & 0.25 & 92.59 & 1.17 & 94.44 & 0.19 \\
\hline 9 & 99.86 & 0.16 & 99.32 & 0.21 & 96.30 & 0.32 & 95.07 & 0.76 \\
\hline
\end{tabular}

Table 6 AMCD and VR on the Lamp iris database

\begin{tabular}{|c|c|c|}
\hline Different methods & AMCD & VR \\
\hline Gabor1+LDA & 0.2612 & 0.0298 \\
\hline Gabor2+LDA & 0.2834 & 0.0306 \\
\hline CPL & 0.6588 & 0.2977 \\
\hline Haar & 0.2937 & 0.1401 \\
\hline
\end{tabular}

recognition rate of the proposed method can reach $98.87 \%$ and it has a lower variance. From Table 4, we can see that the proposed method can classify the iris more efficiently and the performance of the proposed method is stable in terms of VR.

\subsection{Experiment on CASIA-Iris-Lamp Database}

The CASIA-Iris-Lamp database contains right and left eye images of 411 classes, each has 10 iris images and the size of each image is $480 \times 640$ and we choose the left eye images in this Letter. The example images of 2 persons, 5 images per person are shown in Fig. 8. The comparison results are shown in Tables 5 and 6 . From Tables 5 and 6 we can see the performance of the proposed method based on local Gabor orientation feature extraction is better and has a highest robustness.

\section{Conclusion}

In this letter, an iris recognition method based on local Gabor orientation feature extraction is proposed. Experiments are done on the CASIA-Iris-Syn and CASIA-IrisLamp datasets. The experimental results show that the iris orientation features are extracted fully, so the proposed approach can achieve better recognition results than CPL [6] and Haar [7]. The performance of the proposed approach is also consistent. In addition, the constructed local Gabor filters can effectively reduce the feature dimension and feature extraction time compared with Gabor2. But the feature extraction time is still longer than Haar wavelet. We will focus on how to further reduce the feature extraction time and feature dimension in the next work.

\section{Acknowledgments}

This work was partially supported by the Project of Shandong Province Higher Educational Science and Technology Program J14LN25.

\section{References}

[1] C. Liu and M. Xie, "Iris recognition based on DLDA," Proc. 18th Int. Conf. on Pattern Recognition (ICPR'06), Hong Kong, vol.4, pp.489-492, Aug. 2006

[2] P.S. Patil, S.R. Kolhe, R.V. Patil, and P.M. Patil, "The Comparison of Iris Recognition using Principal Component Analysis, Log Gabor and Gabor Wavelets," International Journal of Computer Applications, vol.43, no.1, pp.29-33, April 2012.

[3] A. Ahamed and M.I.H. Bhuiyan, "Low complexity iris recognition using Curvelet transform," Proc. 2012 Int. Conf. on Informatics, Electronics\& Vision (ICIEV), Dhaka, The Bangladesh, pp.548-553, May 2012.

[4] M.R. Rajput and L.M. Waghmare, "Iris feature extraction and recognition based on different transforms," International Journal of Advance in Science Engineering and Technology, vol.1, no.1, pp.3741, July 2013.

[5] W.Q. Yuan, Q. Feng, and L. Ke, "Iris recognition method based on iris texture orientation feature extracted by 2D-Gabor filter," Application Research of Computers, vol.26, no.8, pp.3166-3168, Aug. 2009.

[6] J. Sun, Z.M. Lu, and L.J. Zhou, "Iris recognition using Curvelet transform based on principal component analysis and linear discriminant analysis," Journal of Information Hiding and Multimedia Signal Processing, vol.5, no.3, pp.567-573, July 2014.

[7] T.W. Ng, T.L. Tay, and S.W. Khor, "Iris recognition using rapid Haar wavelet decomposition," Proc. 2nd Int. Conf. on Signal Processing System, Dalian, The China, vol.1, pp.V1-820-V1-823, May 2010.

[8] J.G. Daugman, "High confidence visual recognition of persons by a test of statistical independence," IEEE Trans. Pattern Anal. Mach. Intell., vol.15, no.11, pp.1148-1161, Nov. 1993.

[9] J.R. Sekar, S. Arivazhagan, and R.A. Murugan, "Methodology for iris segmentation and recognition using multi-resolution transform," Proc. Third Int. Conf. on Advanced Computing, pp.82-87, Chennai, 
The Indian, Dec. 2011.

[10] T. Andrysiak and M. Choraś, "Image retrieval based on hierarchical Gabor filters," International Journal of Mathematics and Computer Science, vol.15, no.4, pp.471-480, Jan. 2005.
[11] T.F. Xu, G. WEI, and G.Q. Ni, "Gabor wavelet neural network algorithm based on parallel structure," Optics and Precision Engineering, vol.14, no.2, pp.247-250, April 2006. 